\title{
Decrease in Amplitude of Intracardiac Ventricular Electrogram and Inappropriate Therapy in Patients With an Implantable Cardioverter Defibrillator
}

\author{
Hiroshi Watanabe,,${ }^{1,2}$ MD, Masaomi CHInUShi, ${ }^{3} \mathrm{MD}$, Daisuke IzUMI, ${ }^{2} \mathrm{MD}$, \\ Akinori SATO, ${ }^{2}$ MD, Shinsuke OKADA, ${ }^{2}$ MD, Kazuki OKAMURA, ${ }^{2}$ MD, \\ Satoru Komura, ${ }^{2}$ MD, Yukio HosAKA, ${ }^{2}$ MD, Hiroshi Furushima, ${ }^{2}$ MD, \\ Takashi WASHIZUKA, ${ }^{2} \mathrm{MD}$, and Yoshifusa AIZAWA, ${ }^{2} \mathrm{MD}$
}

\begin{abstract}
SUMMARY
Intracardiac electrograms are important for discrimination of tachyarrhythmia by implantable cardioverter defibrillators (ICD). A low R-wave can cause not only undersensing of ventricular tachyarrhythmia but also inappropriate discharges due to oversensing of unexpected signals because of its characteristic sensing algorithm. Therefore, this study aimed to investigate adverse events associated with R-wave amplitude. We included 115 consecutive patients followed-up over one year after implantation of a transvenous ICD system. The status of the ICD was checked every 3 months and intracardiac ventricular electrograms were analyzed. The decrease in R-wave amplitude was high in arrhythmogenic hypertrophy cardiomyopathy, arrhythmogenic right ventricular cardiomyopathy (ARVC), and sarcoidosis. Low R-waves $(<5.0 \mathrm{mV})$ were observed in 13 patients at a follow-up of $15 \pm 16$ months after implantation, and the mean R-wave was $3.0 \pm 0.8 \mathrm{mV}$. The frequency of low R-waves was high in ARVC (38\%), sarcoidosis (33\%), and dilated cardiomyopathy $(17 \%)$. All of the dilated cardiomyopathy patients with low R-waves had severe left ventricular dysfunction. Inappropriate ICD therapy resulting from T-wave oversensing occurred in 7 patients and the R-wave was $<5.0 \mathrm{mV}$ in 6 of the patients. The frequency of inappropriate therapy was high in patients with sarcoidosis. In 3 patients, inappropriate therapy caused ventricular tachyarrhythmia. In conclusion, decreases in Rwave amplitude occurred in some progressive cardiac disorders and caused inappropriate ICD discharges having arrhythmogenicity. Physicians should attempt to obtain a high Rwave amplitude during ICD implantation and careful follow-up is required, especially in patients with ARVC or sarcoidosis. (Int Heart J 2006; 47: 363-370)
\end{abstract}

Key words: Intracardiac electrogram, Defibrillator, Ventricular tachyarrhythmia, Sarcoidosis, Arrhythmogenic right ventricular cardiomyopathy

From the ${ }^{1}$ Division of Clinical Pharmacology, Vanderbilt University School of Medicine, TN, USA and ${ }^{2}$ Division of Cardiology and ${ }^{3}$ School of Health Sciences, Niigata University Graduate School of Medical and Dental Science, Niigata, Japan. Address for correspondence: Hiroshi Watanabe, MD, Division of Clinical Pharmacology, Vanderbilt University School of Medicine, 2215B Garland Avenue, Room 1275, Nashville, TN 37232-0575, USA.

Received for publication January 16, 2006.

Revised and accepted February 2, 2006. 
IMPLANTABLE cardioverter defibrillators (ICD) effectively terminate ventricular tachyarrhythmia (VTA) and reduce the risk of sudden cardiac death. ${ }^{1-3)}$ ICD can discriminate ventricular tachyarrhythmia from the cycle length and amplitude of intracardiac electrograms with its algorithm for sensing, and thus a decrease in the R-wave may be a cause of undersensing of VTA. Furthermore, as the thresholds of sensing are defined automatically by the amplitude of an R-wave in the algorithm, in the setting of the low R-wave, unfavorable sensing of some signals other than R-wave, eg, T-wave, may occur and can induce inappropriate therapy. ${ }^{4,5}$ Therefore, the amplitude of the intracardiac R-wave is very important in patients with ICD.

Patients with VTA often have diverse types of cardiac disease and low cardiac function. ${ }^{6}$ Some disorders, such as idiopathic cardiomyopathy and inflammatory cardiac disease, can exacerbate progressively. ${ }^{7-9)}$ Myocardial disease or changes in cardiac status may influence R-wave amplitude and prevent adequate sensing of R-wave by ICD. Although low R-wave amplitude has been reported in patients with arrhythmogenic right ventricular cardiomyopathy, ${ }^{10)}$ it is not fully understood in other cardiac diseases. Therefore, the present study was performed to investigate the course of R-wave amplitude and the related adverse events in long-term follow-up in patients with various cardiac diseases.

\section{METHODS}

Patients: The study population included 115 consecutive patients ( 85 men and 30 women) who underwent ICD implantation with a transvenous lead system at Niigata University for the management of life-threatening VTA. The mean age of the patients was $57.6 \pm 14.6$ years. The mean left ventricular ejection fraction was $46.9 \pm 16.5 \%$. The underlying heart diseases were: ischemic heart disease (IHD) in 37 patients, idiopathic ventricular fibrillation (IVF) including Brugada syndrome in 26 patients, dilated cardiomyopathy (DCM) in 18 patients, cardiac sarcoidosis in 9 patients, hypertrophic cardiomyopathy in 8 patients (HCM), arrhythmogenic right ventricular cardiomyopathy (ARVC) in 8 patients, and other cardiac diseases in 9 patients (postoperative state of congenital heart disease in 3, valvular heart disease in 2, idiopathic left ventricular aneurysm in 2, and myotonic dystrophy in 2). The clinical characteristics of the patients are shown in Table I.

ICD implantation: An ICD (model 7223, 7227, or 7271, Medtronic Inc., Minneapolis, MN) was implanted subcutaneously with a transvenous lead system. In all patients, a screw-in lead (model 6936, 6940, and 6943, Medtronic Inc.) was used. The lead was placed in the right ventricular apex, and the sensing and pacing functions were tested and optimized with the following criteria: amplitude of R- 
Table I. Characteristics of Patients

\begin{tabular}{lccccccc}
\hline & $\begin{array}{c}\text { IHD } \\
(n=37)\end{array}$ & $\begin{array}{c}\text { IVF } \\
(n=26)\end{array}$ & $\begin{array}{c}\text { DCM } \\
(n=18)\end{array}$ & $\begin{array}{c}\text { HCM } \\
(n=8)\end{array}$ & $\begin{array}{c}\text { ARVC } \\
(n=8)\end{array}$ & $\begin{array}{c}\text { Sarcoidosis } \\
(n=9)\end{array}$ & $\begin{array}{c}\text { Others } \\
(n=9)\end{array}$ \\
\hline Female (\%) & $10(27 \%)$ & $6(23 \%)$ & $3(17 \%)$ & $0(0 \%)$ & $2(25 \%)$ & $4(44 \%)$ & $5(56 \%)$ \\
Age (years) & $64.9 \pm 9.0$ & $48.5 \pm 19.8$ & $55.4 \pm 13.2$ & $56.1 \pm 9.6$ & $60.0 \pm 9.7$ & $53.6 \pm 15.2$ & $61.0 \pm 10.0$ \\
EF (\%) & $37.7 \pm 13.5$ & $64.4 \pm 5.6$ & $32.4 \pm 9.8$ & $57.9 \pm 17.4$ & $60.8 \pm 8.9$ & $42.3 \pm 6.6$ & $46.0 \pm 13.7$ \\
\hline
\end{tabular}

IHD indicates ischemic heart disease; IVF, idiopathic ventricular fibrillation; DCM, dilated cardiomyopathy; $\mathrm{HCM}$, hypertrophic cardiomyopathy; ARVC, arrhythmogenic right ventricular cardiomyopathy; and EF, ejection fraction of the left ventricle. Others includes congenital heart disease $(n=4)$, valvular heart disease $(n=2)$, idiopathic left ventricular aneurysm $(n=2)$, and myotonic dystrophy $(n=2)$.

wave $>5.0 \mathrm{mV}$ and pacing threshold of $<1.5 \mathrm{~V}$ per $0.5 \mathrm{~ms}$. Ventricular sensing was set at $\geq 0.3 \mathrm{mV}$.

Follow-up: The status of the ICD and episodes of ICD therapy were followed-up 2 weeks after implantation and every 3 months thereafter at the outpatient clinic. In all patients, electrocardiography and chest $\mathrm{x}$-rays were performed at every visit to the outpatient clinic. The $\mathrm{R}$ wave amplitude was measured from the telemetered intracardiac electrograms from the ICD.

Data and statistical analysis: We compared R-wave amplitude among underlying cardiac diseases, as well as the number of patients with a low R-wave amplitude defined as $<5 \mathrm{mV}$ and inappropriate ICD therapies associated with low R-wave amplitude. The statistical significance of differences was tested by ANOVA or Student's $t$-test. Data are expressed as the mean $\pm \mathrm{SD}$. Differences were considered significant at $P<0.05$.

\section{RESULTS}

Decrease in R-wave: In all patients, the mean R-wave amplitude was similar between $10.9 \pm 3.9 \mathrm{mV}$ at ICD implantation and $10.8 \pm 4.1 \mathrm{mV} 3$ months after implantation. During a follow-up of $59 \pm 31$ months, the R-wave decreased slightly to $9.0 \pm 3.8 \mathrm{mV}$ but this was not significant $(P=0.40)$, and the percent decrease in the R-wave was $9.0 \pm 3.8 \%$. The decrease in the R-wave was high in HCM, ARVC, and sarcoidosis during the follow-up (Table II). The follow-up periods were not different among the cardiac disease groups.

Low R-waves $(<5.0 \mathrm{mV})$ occurred in 13 patients at a follow-up of $15 \pm 16$ months (range, 0-48 months) after ICD implantation, and the mean R-wave was $3.0 \pm 0.8 \mathrm{mV}$ (range, 1.5-4.0 mV, Table III). In one patient with sarcoidosis (case 12), a sufficient $\mathrm{R}$-wave amplitude was not obtained during implantation despite detailed mapping of the right ventricle and the lead was placed at the site of a low 
Table II. Incidence of Low R-wave

\begin{tabular}{|c|c|c|c|c|c|c|c|}
\hline & IHD & IVF & DCM & $\mathrm{HCM}$ & ARVC & Sarcoidosis & Others \\
\hline \multicolumn{8}{|l|}{ R-wave (mV) } \\
\hline Implantation & $11.4 \pm 3.2$ & $9.7 \pm 2.3$ & $12.1 \pm 4.7$ & $12.6 \pm 6.0$ & $9.4 \pm 4.4$ & $7.6 \pm 4.4$ & $12.4 \pm 3.0$ \\
\hline 3 months & $11.0 \pm 4.0$ & $10.7 \pm 2.9$ & $9.6 \pm 4.1$ & $14.2 \pm 6.5$ & $9.6 \pm 4.2$ & $9.9 \pm 5.9$ & $12.0 \pm 2.4$ \\
\hline Minimum & $9.8 \pm 3.8$ & $8.9 \pm 2.6$ & $8.4 \pm 3.6$ & $10.4 \pm 4.7$ & $7.0 \pm 5.1$ & $7.9 \pm 5.0$ & $10.1 \pm 3.0$ \\
\hline Decrease $(\mathrm{mV})$ & $1.1 \pm 1.7$ & $1.8 \pm 2.6$ & $1.2 \pm 1.5$ & $3.8 \pm 3.5$ & $2.6 \pm 2.2$ & $2.2 \pm 2.1$ & $1.9 \pm 1.4$ \\
\hline$\%$ Decrease & $10.3 \pm 14.7$ & $14.6 \pm 19.7$ & $11.4 \pm 12.8$ & $22.7 \pm 21.5$ & $32.5 \pm 27.6$ & $22.9 \pm 20.1$ & $17.5 \pm 16.3$ \\
\hline Low R-wave (N) & $2(5 \%)$ & $1(4 \%)$ & $3(17 \%)$ & $0(0 \%)$ & $3(38 \%)$ & $3(33 \%)$ & $1(11 \%)$ \\
\hline Inappropriate therapy* $(\mathrm{N})$ & $0(0 \%)$ & $1(4 \%)$ & $2(11 \%)$ & $1(13 \%)$ & $0(0 \%)$ & $3(33 \%)$ & $0(0 \%)$ \\
\hline
\end{tabular}

The minimum R-wave was defined as the lowest R-wave during follow-up. The decrease in R-wave amplitude was calculated as the difference between the R-wave at 3 months after the implantation and the minimum R-wave. An R-wave $<5.0 \mathrm{mV}$ was considered low amplitude. See Table I for abbreviations.

* Inappropriate therapy due to T-wave oversensing.

Table III. Characteristics of Patients With a Low R-wave

\begin{tabular}{ccccccc}
\hline \multirow{2}{*}{$\begin{array}{c}\text { Patient } \\
\text { No. }\end{array}$} & $\begin{array}{c}\text { Sex/Age } \\
\text { (years) }\end{array}$ & Heart disease & \multicolumn{2}{c}{ R-wave $(\mathrm{mV})$} & $\begin{array}{c}\text { Time at } \\
\text { R-wave }<5 \mathrm{mV}\end{array}$ & $\begin{array}{c}\text { Inappropriate } \\
\text { therapy* }\end{array}$ \\
\cline { 4 - 5 } & & Implantation & Minimum & & No \\
\hline 1 & $\mathrm{M} / 57$ & ARVC & 5.0 & 3.0 & $12 \mathrm{M}$ & No \\
3 & $\mathrm{~F} / 39$ & ARVC & 5.5 & 3.0 & $6 \mathrm{M}$ & No \\
4 & $\mathrm{M} / 62$ & ARVC & 6.5 & 3.5 & $3 \mathrm{M}$ & No \\
5 & $\mathrm{M} / 70$ & CHD & 16.0 & 3.5 & $36 \mathrm{M}$ & No \\
6 & $\mathrm{~F} / 30$ & DCM & 8.0 & 3.0 & $3 \mathrm{M}$ & Yes \\
7 & $\mathrm{M} / 74$ & DCM & 8.6 & 3.5 & $6 \mathrm{M}$ & Yes \\
8 & $\mathrm{M} / 56$ & IVF & 7.0 & 4.0 & $3 \mathrm{M}$ & Yes \\
9 & $\mathrm{~F} / 70$ & IHD & 10.0 & 3.0 & $48 \mathrm{M}$ & No \\
10 & $\mathrm{M} / 49$ & IHD & 9.0 & 4.0 & $24 \mathrm{M}$ & No \\
11 & $\mathrm{~F} / 63$ & Sarcoidosis & 7.0 & 1.5 & $6 \mathrm{M}$ & Yes \\
12 & $\mathrm{~F} / 64$ & Sarcoidosis & 4.0 & 1.5 & Implantation & Yes \\
13 & $\mathrm{M} / 26$ & Sarcoidosis & 9.0 & 3.5 & $36 \mathrm{M}$ & Yes \\
\hline
\end{tabular}

* Inappropriate therapy due to T-wave oversensing.

R-wave $(4.0 \mathrm{mV})$. However, in the other 12 patients, the R-wave amplitude at ICD implantation met the criteria for implantation. The frequency of a low Rwave was high in ARVC (38\%), sarcoidosis (33\%), and DCM (17\%). All 3 DCM patients with a low R-wave had severe left ventricular dysfunction at the baseline (ejection fraction $\leq 30 \%$ ). None of the patients presented a low R-wave with lead fracture, significant change in lead impedance or pacing thresholds, or worsening of heart disease or congestive heart failure. The surface electrocardiogram did not change during the follow-up period in any patient with a low R-wave. The low Rwave did not occur with increasing activity of sarcoidosis as determined by blood tests, including angiotensin-converting enzyme and lysozyme, and gallium scin- 


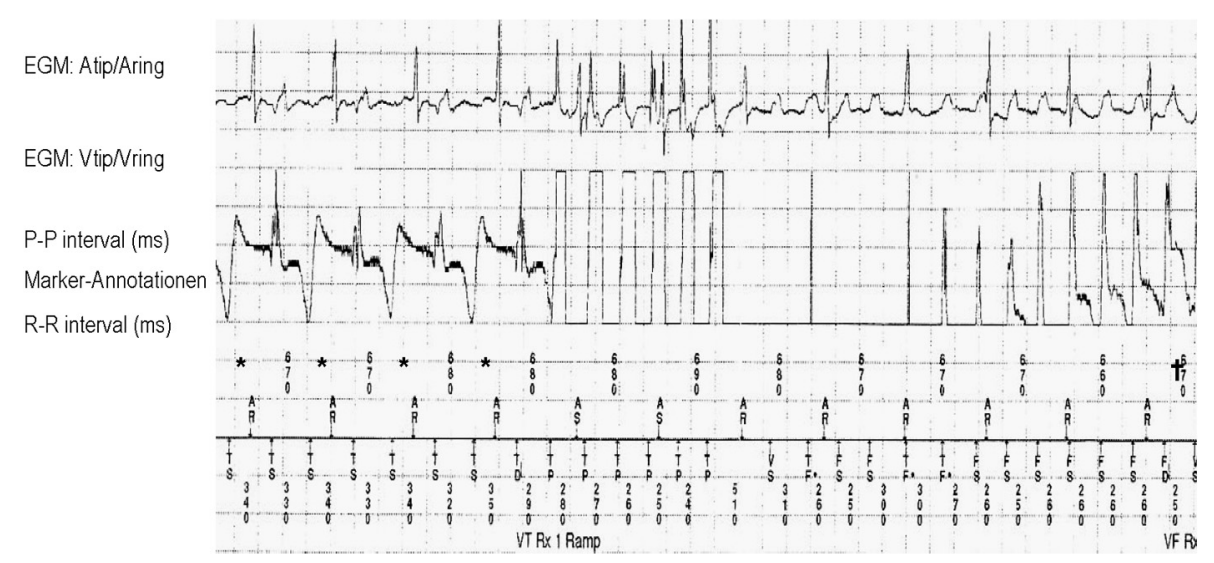

Figure. Inappropriate therapy-induced ventricular tachyarrhythmia.

Due to T-wave oversensing $(*)$ during sinus rhythm, inappropriate antitachycardia pacing was delivered. The inappropriate therapy induced ventricular tachyarrhythmia detected as ventricular fibrillation $(\dagger)$ by an implantable cardioverter defibrilla-

tigraphy in any of the patients. There was no undersensing of ventricular tachyarrhythmia in patients with a low R-wave.

Inappropriate therapy associated with low R-wave: Inappropriate ICD therapy resulting from T-wave oversensing occurred in 7 patients at $16.7 \pm 19.3$ months after implantation (range, 1-47 months). The number of occurrences of inappropriate therapy due to T-wave oversensing was 6.9 \pm 6.8/person (range, 2-20). In 6 of the 7 patients, an R-wave of $<5.0 \mathrm{mV}$ in an intracardiac electrogram was recorded when the therapy was inappropriate, and the low $\mathrm{R}$-wave was confirmed thereafter at the outpatient clinic. In the remaining patient with HCM, the R-wave amplitude was $12.0 \mathrm{mV}$ when inappropriate therapy was undertaken due to the spontaneous increase in the T-wave. The frequency of inappropriate therapy due to T-wave oversensing was high in patients with sarcoidosis (33\%). In 3 patients, inappropriate therapy induced ventricular tachyarrhythmia, although all cases of tachyarrhythmia terminated spontaneously or by ICD (Figure). In 4 of the 6 patients with inappropriate therapy associated with a low R-wave (patients 6,8 , 11 , and 12), inappropriate therapy recurred after changing the algorithm for tachyarrhythmia detection, changing the pacing rate, and/or administration of betablocking agents. Finally, in 2 patients (patients 6 and 8), a sensing lead was added to the septum of the right ventricle, and inappropriate therapy did not occur thereafter. 


\section{DISCUSSION}

A decrease in R-wave amplitude occurs frequently in cardiac sarcoidosis and arrhythmogenic right ventricular cardiomyopathy. A low R-wave could result in inappropriate ICD therapy, which may trigger ventricular tachyarrhythmia.

Sarcoidosis is a systemic granulomatous disease of unknown cause affecting any organ. ${ }^{11-13)}$ Cardiac involvement is progressively and potentially fatal, although uncommon. ARVC is characterized by replacement of the myocardium by fatty and fibrous tissue, and myocardial atrophy predominantly in the right ventricle is also progressive. ${ }^{8,14,15)}$ The high frequency of large decreases in Rwave amplitude in sarcoidosis and ARVC may be associated with progression of myocardial invasion to the right ventricle, but it was not shown by conventional examination. Blood tests did not reveal an increase in sarcoidosis. Magnetic resonance imaging, which is useful for evaluating cardiac involvement in ARC, could not be performed because of controversy around ICD. The local myocardium may be easily affected by mechanical stress, such as lead placement, in such progressive disorders.

Low R-waves were also found in patients with DCM and severe left ventricular dysfunction. Dilated cardiomyopathy is also a progressive disease of the myocardium, which is characterized by predominant enlargement of the left ventricle, decreased systolic function, and reduced myocardial wall thickness. ${ }^{8,9)}$ The reduction in the number and function of myocardial fibers leads to interstitial fibrosis. ${ }^{9)}$ Although the main focus has traditionally been placed on assessment of the left ventricle, right ventricular dysfunction and thinning of the right ventricular wall have also been reported, even in the well-controlled state. ${ }^{16)}$ Moreover, advanced left ventricular dysfunction can increase right ventricular overload and may cause extensive fibrosis, possibly resulting in a decrease in R-wave. Although worsening of heart failure might influence R-wave amplitude, a low Rwave amplitude was not found after development of heart failure in any patients.

A low R-wave can cause not only undersensing of ventricular tachyarrhythmia, but also inappropriate therapy associated with T-wave oversensing because of the characteristic automatic algorithm of ICD for sensing. In the present study, approximately $46 \%$ of patients with a low R-wave experienced inappropriate therapy having arrhythmogenicity. In the setting of a low R-wave amplitude, an increase in sensing threshold to avoid T-wave oversensing can increase the risk of undersensing of ventricular tachyarrhythmia. Pharmacological therapy to affect $\mathrm{R}$-wave or T-wave amplitude may be difficult. To avoid inappropriate therapy, we changed the ICD parameters and administered beta-blocking agents to slow sinus rhythm. However, inappropriate therapy occurred repeatedly in most of the 
patients. Therefore, addition of a sensing lead may be the only reliable way to resolve the problem. Moreover, because the ventricular apex is one of the areas frequently involved in ARVC and sarcoidosis, lead replacement to another area might prevent the reappearance of decreasing R-waves. ${ }^{17,18)}$ However, the diseased right ventricular myocardium may prevent adequate lead replacement, and low R-wave may develop again.

Limitations: The number of patients with each disorder was relatively small and our observations will need to be confirmed in a larger population, especially in patients with sarcoidosis, before they can be accepted as definitive. As only screw-in leads were used, the outcome of tined leads could not be studied.

Conclusions: The risk of adverse events of ICD associated with R-wave amplitude is high in patients with progressive cardiac disorders, such as ARVC, sarcoidosis, or DCM. Physicians should attempt to obtain as high an R-wave amplitude as possible at lead placement in ICD implantation and carefully monitor the Rwave, especially in patients with these disorders.

\section{REFERENCES}

1. A comparison of antiarrhythmic-drug therapy with implantable defibrillators in patients resuscitated from nearfatal ventricular arrhythmias. The Antiarrhythmics versus Implantable Defibrillators (AVID) Investigators. N Engl J Med 1997; 337: 1576-83.

2. Kuck KH, Cappato R, Siebels J, Ruppel R. Randomized comparison of antiarrhythmic drug therapy with implantable defibrillators in patients resuscitated from cardiac arrest: the Cardiac Arrest Study Hamburg (CASH). Circulation 2000; 102: 748-54.

3. Connolly SJ, Gent M, Roberts RS, et al. Canadian implantable defibrillator study (CIDS) : a randomized trial of the implantable cardioverter defibrillator against amiodarone. Circulation 2000; 101: 1297-302.

4. Washizuka T, Chinushi M, Kasai H, et al. Inappropriate discharges from an intravenous implantable cardioverter defibrillator due to T-wave oversensing. Jpn Circ J 2001; 65: 685-7.

5. Hsu SS, Mohib S, Schroeder A, Deger FT. T wave oversensing in implantable cardioverter defibrillators. J Interv Card Electrophysiol 2004; 11: 67-72.

6. Watanabe H, Chinushi M, Washizuka T, et al. Electrophysiologic study-guided therapy with sotalol for lifethreatening ventricular tachyarrhythmias. Pacing Clin Electrophysiol 2005; 28: 285-90.

7. Newman LS, Rose CS, Maier LA. Sarcoidosis. N Engl J Med 1997; 336: 1224-34. (Review)

8. Richardson P, McKenna W, Bristow M, et al. Report of the 1995 World Health Organization/International Society and Federation of Cardiology Task Force on the Definition and Classification of Cardiomyopathies. Circulation 1996; 93: 841-2.

9. Hughes SE, McKenna WJ. New insights into the pathology of inherited cardiomyopathy. Heart 2005; 91: $257-$ 64. (Review)

10. Wichter T, Paul M, Wollmann C, et al. Implantable cardioverter/defibrillator therapy in arrhythmogenic right ventricular cardiomyopathy: single-center experience of long-term follow-up and complications in 60 patients. Circulation 2004; 109: 1503-8.

11. Flora GS, Sharma OP. Myocardial sarcoidosis: a review. Sarcoidosis 1989; 6: 97-106. (Review)

12. Sharma OP. Sarcoidosis. Dis Mon 1990; 36: 469-535. (Review)

13. Shammas RL, Movahed A. Sarcoidosis of the heart. Clin Cardiol 1993; 16: 462-72. (Review)

14. Thiene G, Nava A, Corrado D, Rossi L, Pennelli N. Right ventricular cardiomyopathy and sudden death in young people. N Engl J Med 1988; 318: 129-33. 
15. Fontaine G, Fontaliran F, Frank R. Arrhythmogenic right ventricular cardiomyopathies: clinical forms and main differential diagnoses. Circulation 1998; 97: 1532-5. (Review)

16. Oyama S, Sakuma M, Komaki K, et al. Right ventricular systolic function and the manner of transformation of the right ventricle in patients with dilated cardiomyopathy. Circ J 2004; 68: 933-7.

17. van der Wall EE, Kayser HW, Bootsma MM, de Roos A, Schalij MJ. Arrhythmogenic right ventricular dysplasia: MRI findings. Herz 2000; 25: 356-64. (Review)

18. Le Guludec D, Slama MS, Frank R, et al. Evaluation of radionuclide angiography in diagnosis of arrhythmogenic right ventricular cardiomyopathy. J Am Coll Cardiol 1995; 26: 1476-83. 